\title{
Entanglement in the bimodal Jaynes-Cummings model with the two-mode squeezed vacuum state
}

\author{
Faisal A. A. El-Orany* \\ Cyberspace Security Laboratory, MIMOS Berhad, \\ Technology Park Malaysia, 57000 Kuala Lumpur, \\ Malaysia; Department of Mathematics and Computer Science, \\ Faculty of Science, Suez Canal University, Ismailia, Egypt; \\ S. Abdel-Khalek, M. Abdel-Aty, and M. R. B. Wahiddin \\ Cyberspace Security Laboratory, MIMOS Berhad, \\ Technology Park Malaysia, 57000 Kuala Lumpur, Malaysia
}

\begin{abstract}
In this paper, we study the interaction between the two-level atom and a bimodal cavity field, namely, two-mode Jaynes-Cummings model when the atom and the modes are initially in the atomic superposition state and two-mode squeezed vacuum state, respectively. For this system we investigate the atomic inversion, linear entropy and atomic Wehrl entropy. We show that there is a connection between all these quantities. Also we prove that the atomic Wehrl entropy exhibits behaviors similar to those of the linear entropy and the von Neumann entropy. Moreover, we show that the bipartite exhibits periodical disentanglement and derive the explicit forms of the states of the atom and the modes at these values of the interaction times.

PACS numbers: 42-50.Dv Jaynes-Cummings model, atomic inversion, two-mode squeezed vacuum states, Wehrl entropy, linear entropy.
\end{abstract}

\section{INTRODUCTION}

One of the few exactly solvable models in quantum optics, which describes the interaction between the radiation field and the matter, is the JaynesCummings model (JCM) [1]. The JCM has become experimentally realizable with the Rydberg atoms in the high-Q microwave cavities (e.g., see [2]). Various extensions for the JCM have been performed and investigated in greater details such as multiphoton [3, 4], intensity-dependent [5], multimode, e.g., [6, 7, 8, 9, 10], multilevel atoms [11] and multiatom interactions [12]. One of these extensions is the two-mode JCM (TJCM), which has taken a considerable interest in the literatures, e.g. [6, 7, 9, 10, 13, 14]. The revival-collapse phenomenon (RCP) occurring in the atomic inversion of the TJCM is rather complicated compared to that of the standard JCM (, i.e. a single mode interacting with a single atom) in the sense that the revival series is compact and each revival is followed with a secondary revival. Furthermore, the RCP associated with the TJCM is independent of the initial intensities of the modes. Such behavior has been partially explained in [9], however, an investigation for the occurrence of the secondary revivals is

*Electronic address: el’orany@yahoo.com 
given in [6]. The quantum phase properties for the TJCM with the initial Schrödinger-cat states have been investigated in [7] showing that the phase variances of the single-mode case can exhibit RCP about the long-time behavior but with the interaction time several times smaller than that of the standard JCM. Furthermore, there is a clear relationship between the RCP occurring in the atomic inversion and the behavior of the phase distribution of both the single-mode and the two-mode cases. Moreover, for the TJCM it has been shown that there is a class of states for which the quadratures squeezing exhibit RCP similar to that involved in the corresponding atomic inversion [13].

Quantum information processing provides different way for manipulating information than the classical one. This is related to entanglement, which plays an essential role in the quantum information [15] such as quantum computing [16], teleportation [17], cryptographic [18], dense coding [19] and entanglement swapping [20]. Thus intensive efforts have been done to understand theoretically and experimentally the entanglement in the quantum systems. For instance, the entanglement between two qubits in an arbitrary pure state has been quantified by the concurrence [21] and Peres-Horodecki measure [22], however, that of the mixed states quantified by the infimum of the average concurrence over all possible pure state ensemble decomposition. Additionally, the entropic relations are used in investigating the entanglement in the quantum system. In this regard von Neuman entropy (NE) [23], linear entropy (LE) and the Shannon information entropy (SE) 24] have been frequently used in treating entanglement in the quantum systems. The NE [25] and the LE [3] have been applied to the JCM. It is worth mentioning that the SE involves only the diagonal elements of the density matrix and in some cases it can give information similar to that obtained from the $\mathrm{NE}$ and LE. On the other hand, there is an additional entropic relation, namely, the Wehrl entropy (WHE) [26]. This relation has been successfully applied in description of different properties of the quantum optical fields such as phase-space uncertainty [27, 28], quantum interference [28], decoherence [29, 30] etc. The WHE is more sensitive in distinguishing states than the NE since WHE is a state dependent [31]. The concept of the Wehrl phase distribution has been developed and shown that it serves as a measure of both noise (phasespace uncertainty) and phase randomization [31]. Furthermore, the WHE has been applied to the dynamical systems, too. In this respect the time evolution of the WHE for the Kerrlike medium has been discussed in [32] showing that the WHE gives a clear signature for the formation of finite superpositions of coherent states (cat-like states) as well as the number of coherent components taking part in the superposition. For the JCM the WHE gives an information on the splitting of the $Q$-function in the course of the collapse region of the atomic inversion as well as on the atomic inversion itself [30, 33]. In the present paper we show that the atomic Wehrl entropy (AHE) [33] can be used to quantify entanglement in the TJCM when the modes are initially prepared in the maximally entangled states such as the two-mode squeezed vacuum state (TMS) [34]. The TMS can be expressed as

$$
|r\rangle=\sum_{n=0}^{\infty} C_{n}|n, n\rangle,
$$

where $C_{n}=\frac{1}{\cosh r} z^{n}, \quad z=\tanh r$. We should stress that the TMSs are important states since they contain quantum correlations between the different modes that make up the field. Based on this fact the TMSs have been used in the continuous-variable teleportation [35], quantum key distribution [36], verification of EPR correlations [37], etc. Most importantly the TMSs can be experimentally generated via the optical parametric oscillator [38], where squeezing can be detected with high efficiency homodyne detector [39]. Now for the TJCM with the 
initial TMS we investigate the atomic inversion, LE, Wehrl atomic density and AHE. This work is motivated by the importance of the TJCM and TMS in quantum optics as well as in the quantum information, as we mentioned above. We obtain many of interesting results such as the atomic Wehrl density (entropy) can give qualitative (quantitative) information on the entanglement in the TJCM. In other words, the AWE can be used as a new measure for quantifying entanglement in the quantum systems. Furthermore, we show that the bipartite (, i.e. two-mode and atom) can periodically exhibit instantaneous (long-lived) disentanglement. Also we derive the explicit forms for the states of the atom and the twomode at these values of the interaction times. The paper is prepared in the following order: In section 2 we derive the wavefunction of the system and evaluate the expectation values of the atomic variables. In section 3 we investigate the atomic inversion and the LE. In section 4 we discuss the atomic Wehrl density and the atomic Wehrl entropy. In section 5 we summarize the main results.

\section{WAVEFUNCTION OF THE SYSTEM}

In this section we derive the dynamical wavefunction for the system, which consists of two-mode field interacting with the two-level atom. Also, we evaluate the expectation values for the atomic operators which will be frequently used in the paper.

The Hamiltonian describing the interaction between two-mode field and the two-level atom in the rotating wave approximation takes the form [40]:

$$
\hat{H}=\hbar\left(\hat{H}_{0}+\hat{H}_{i}\right),
$$

where

$$
\begin{aligned}
& \hat{H}_{0}=\sum_{j=1}^{2} \omega_{j} \hat{a}_{j}^{\dagger} \hat{a}_{j}+\omega_{a} S_{z}, \\
& \hat{H}_{i}=\chi\left(\hat{a}_{1}^{\dagger 2} \hat{a}_{1}^{2}+\hat{a}_{2}^{\dagger 2} \hat{a}_{2}^{2}\right)+\lambda\left[\hat{a}_{1} \hat{a}_{2} \hat{S}_{+}+\hat{a}_{1}^{\dagger} \hat{a}_{2}^{\dagger} \hat{S}_{-}\right],
\end{aligned}
$$

where $\hat{a}_{j} \quad\left(\hat{a}_{j}^{\dagger}\right)$ and $\omega_{j}$ are the annihilation (creation) operator and frequency designated the $j$ th mode, the set $\left\{\hat{S}_{z}, \hat{S}_{+}, \hat{S}_{-}\right\}$is the usual Pauli spin operators, $\lambda$ is the coupling constant between the atom and the modes, $\chi$ is the dispersive part of the third-order nonlinearity of the Kerr-like medium, which is assumed to be the same for the two modes. Also $\omega_{a}$ denotes the atomic transition frequency.

Throughout the investigation we consider that $\omega_{a}=\omega_{1}+\omega_{2}$ (i.e. the resonance case), the modes are initially prepared in the TMS (1) and the atom is in the atomic superposition state having the form:

$$
\left|\Psi_{a}(0)\right\rangle=\cos \left(\frac{\theta}{2}\right)|\uparrow\rangle+\sin \left(\frac{\theta}{2}\right) \exp (i \phi)|\downarrow\rangle,
$$

where $|\uparrow\rangle$ and $|\downarrow\rangle$ deonte excited and ground atomic states, respectively; $\theta$ and $\phi$ are phases. It is worth reminding that preparing the atom in the coherent superposition states is important as a result of its applications in noise quenching by correlated spontaneous emission [41], quantum beats [42] and noise-free amplification [43]. Now under these conditions the 
dynamical wavefunction for the Hamiltonian system (3) can be evaluated in the standard way as

$$
|\Psi(T)\rangle=\sum_{n=0}^{\infty} \exp \left(-2 i \eta T n^{2}\right)\left[F_{1}(n, T)|\uparrow, n, n\rangle+F_{2}(n, T)|\downarrow, n+1, n+1\rangle\right]
$$

where

$$
\begin{aligned}
& F_{1}(n, T)=C_{n} \cos \frac{\theta}{2} \cos \left(T \Omega_{n}\right)+\frac{i C_{n}}{\Omega_{n}}\left[2 \eta n \cos \frac{\theta}{2}-(n+1) \exp (i \phi) \sin \frac{\theta}{2}\right] \sin \left(T \Omega_{n}\right) \\
& F_{2}(n, T)=C_{n} \exp (i \phi) \sin \frac{\theta}{2} \cos \left(T \Omega_{n}\right)-\frac{i C_{n}}{\Omega_{n}}\left[2 \eta n \exp (i \phi) \sin \frac{\theta}{2}+(n+1) \cos \frac{\theta}{2}\right] \sin \left(T \Omega_{n}\right)
\end{aligned}
$$

and

$$
\Omega_{n}=\sqrt{4 \eta^{2} n^{2}+(n+1)^{2}}, \quad \eta=\frac{\chi}{\lambda}, \quad T=t \lambda .
$$

All information about the system is involved in the wavefunction (5) or in the total density matrix $\hat{\rho}(T)=|\Psi(T)\rangle\langle\Psi(T)|$. As we mainly deal with the atomic subsystem we evaluate the atomic reduced density matrix $\hat{\rho}_{a}(T)$ via the relation:

$$
\hat{\rho}_{a}(T)=\operatorname{Tr}_{f} \hat{\rho}(T)
$$

where the subscript $f$ means that the trace is taken over the field. We close this section by evaluating the expectation values for the atomic operators $\left\{\hat{S}_{z}, \hat{S}_{x}, \hat{S}_{y}\right\}$ for (5) as

$$
\begin{aligned}
& \left\langle\hat{S}_{x}(T)\right\rangle=\frac{1}{2} \rho_{x}(T)=\operatorname{Re} \sum_{n=0}^{\infty} \exp [2 i \eta T(2 n+1)] F_{1}^{*}(n+1, T) F_{2}(n, T), \\
& \left\langle\hat{S}_{y}(T)\right\rangle=\frac{1}{2} \rho_{y}(T)=\operatorname{Im} \sum_{n=0}^{\infty} \exp [2 i \eta T(2 n+1)] F_{1}^{*}(n+1, T) F_{2}(n, T), \\
& \left\langle\hat{S}_{z}(T)\right\rangle=\frac{1}{2} \rho_{z}(T)=\frac{1}{2} \sum_{n=0}^{\infty}\left[\left|F_{1}(n, T)\right|^{2}-\left|F_{2}(n, T)\right|^{2}\right],
\end{aligned}
$$

where $\hat{S}_{x}=\frac{1}{2}\left(\hat{S}_{-}+\hat{S}_{+}\right), \hat{S}_{y}=\frac{1}{2 i}\left(\hat{S}_{+}-\hat{S}_{-}\right)$. We use the results obtained in this section to make a comparative study between the atomic inversion, LE and AWE in the following sections.

\section{ATOMIC INVERSION AND LINEAR ENTROPY}

In this section we investigate the atomic inversion and the LE for the system under consideration. We start the investigation with the atomic inversion, which is plotted in Fig. 1 versus the scaled time $T$ for the given values of the interaction parameters. From this figure the obvious remark is that the $\left\langle\hat{S}_{z}(T)\right\rangle$ is periodic with period $\pi$, regardless of the type of the initial atomic state. The origin in this is related to the nature of the TMS in which the information is equally distributed among the two modes [44]. Furthermore, such periodicity is quite similar to that of the two-photon JCM [4] and intensity-dependent JCM [5]. For the superposition atomic state $(\theta, \phi)=(\pi / 2, \pi / 2)$, i.e. the dashed curve, one can observe that $\left\langle\hat{S}_{z}(T)\right\rangle$ oscillates around zero with extreme values \pm 0.15 . Nevertheless, we have found 


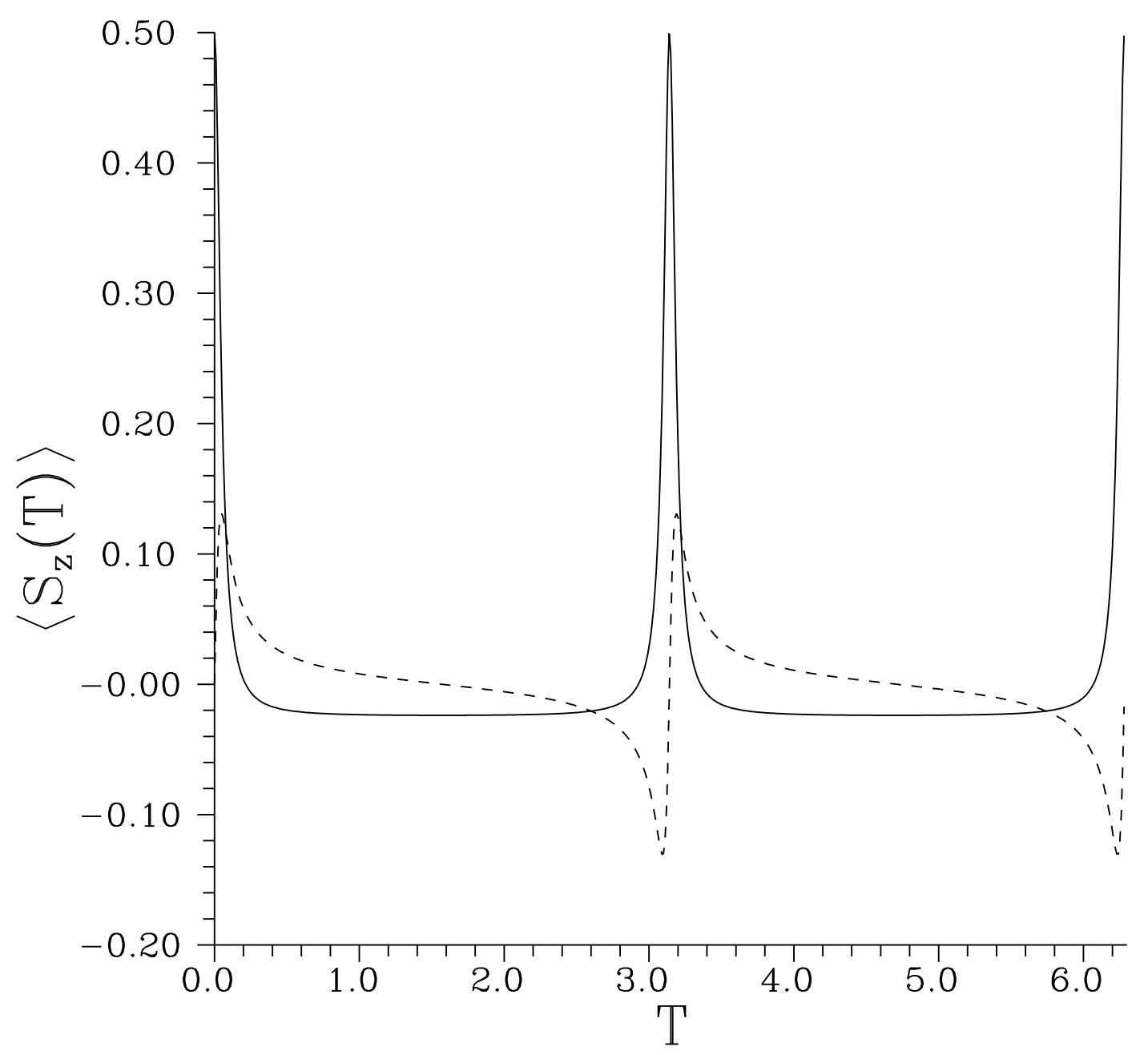

FIG. 1: The $\left\langle\hat{S}_{z}(T)\right\rangle$ versus scaled time $T$ for $\sinh ^{2} r=10$ and $(\theta, \phi, \eta)=(0,0,0)$ (solid curve) and $(\pi / 2, \pi / 6,0)$ (dashed curve).

that the system can exhibit atomic trapping, i.e. $\left\langle\hat{S}_{z}(T)\right\rangle=0$, for $(\theta, \phi)=(\pi / 2,0)$, as we show shortly. All these facts can be analytically realized for $\eta=0$. In this case $\left\langle\hat{S}_{z}(T)\right\rangle$ in (9) can be easily modified as:

$$
\left\langle\hat{S}_{z}(T)\right\rangle=\frac{\left[\cos (2 T)-z^{2}\right] \cos \theta+z \sin \theta \sin (\phi) \sin (2 T)}{2\left[\left(1-z^{2} \cos (2 T)\right)^{2}+z^{4} \sin ^{2}(2 T)\right] \cosh ^{2} r} .
$$

From (10) the smallest value of the atomic inversion for $\theta=0$ is $\left\langle\hat{S}_{z}(T)\right\rangle=-1 /(2+2 \bar{n})$, where $\bar{n}=2 \sinh ^{2} r$, which occurs at $T=\pi / 2$ (, i.e. collapse time). This means that the greater the initial mean-photon number of the field the closer the value of the $\left\langle\hat{S}_{z}(T)\right\rangle$ to zero at the collapse time. Also it is obvious that for $(\theta, \phi)=(\pi / 2,0)$ the expression (10) tends to zero. We have found that the behavior of the $\left\langle\hat{S}_{z}(T)\right\rangle$ is insensitive of the value of $\eta$. Finally the information obtained here is important in investigating the entanglement in the bipartite, as we show below.

Now we quantify the entanglement between the two-mode system and the atomic system 

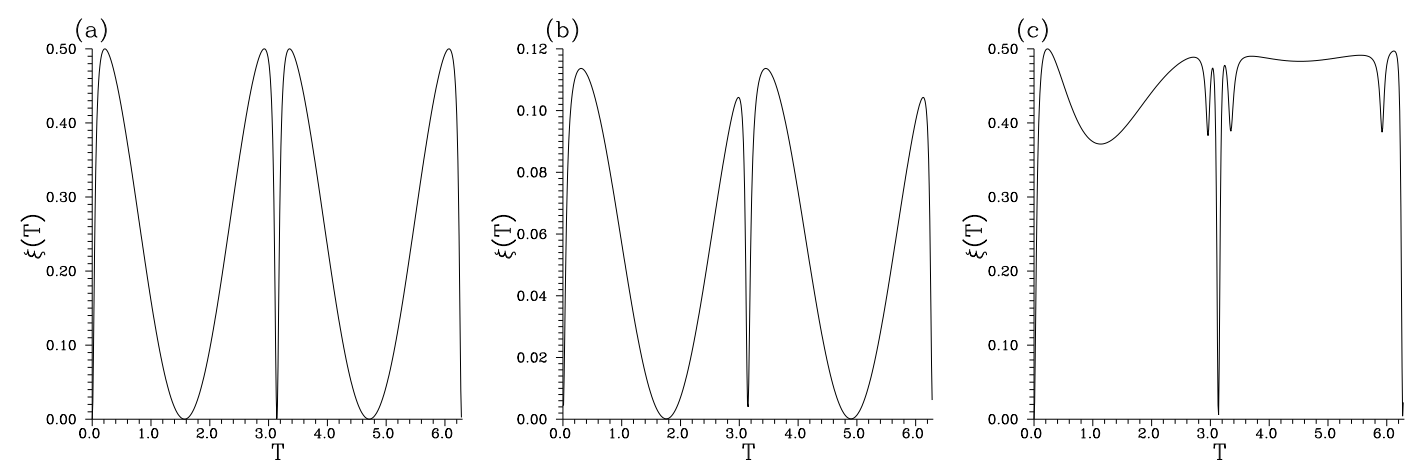

FIG. 2: The $\xi(T)$ versus scaled time $T$ for $\sinh ^{2} r=10$ and $(\theta, \phi, \eta)=(0,0,0)(\mathrm{a}),(\pi / 2, \pi / 6,0)$ (b) and $(0,0,0.03)(\mathrm{c})$.

via the linear entropy, which is defined by

$$
\xi(T)=1-\operatorname{Tr} \hat{\rho}_{a}^{2}(T) .
$$

As a result of the symmetry in the system the linear entropies for the atomic and field sub-systems are equivalent. Furthermore, as the system is in an over all pure state, the LE is a relevant measure for quantifying entanglement in the bipartite [45]. The $\xi(T)$ ranges from 0 for pure states and/or disentangled states to 0.5 for a maximally entangled states [46]. For the future purpose, the relation (11) can be expressed in terms of the expectation values of the atomic variables (9) as

$$
\xi(T)=\frac{1}{2}\left[1-\rho_{x}^{2}(T)-\rho_{y}^{2}(T)-\rho_{z}^{2}(T)\right] .
$$

In Figs. 2 we plot $\xi(T)$ versus the scaled time $T$ for given values of the interaction parameters. From Fig. 2(a) and Fig. 2(b), where $\eta=0$, the bipartite is periodically disentangled with period $\pi$. As we mentioned above this is related to the nature of the TMS. Also this is manifested in the wavefunction (5) as linear Rabi frequency $\Omega_{n}$ in the parameter $n$. Moreover, from Figs. 2(a)-(b) the $\xi(T)=0$ at $T=0$, and when the interaction is switched on, i.e. the interchange of energy between the two modes and atom occurs, the bipartite exhibits immediately maximum entanglement (i.e. $\xi(T)$ provides maximum value), stays for a while then exhibiting long-lived disentanglement at $T=\pi / 2$. As the interaction proceeds, the $\xi(T)$ goes gradually to the maximum value (i.e. entanglement) and eventually tends to zero at $T=\pi$. Furthermore, the comparison between Fig. 2(a) and Fig. 2(b) shows that the atomic coherence decreases the amount of entanglement in the bipartite. This agrees with the fact that the interference in phase space decreases the degree of entanglement in the bipartite [47]. To obtain more accurate information on the disentanglement in the bipartite for $\eta=0$ we give a closer look at the analytical form of the wavefunction (5) at $T=m \pi$ and $T=m^{\prime} \pi / 2$, where $m$ and $m^{\prime}$ are integer and odd integer numbers, respectively. At these values of the interaction times the wavefunction (5) can be easily expressed as:

$$
|\psi(T=m \pi)\rangle=\sum_{n=0}^{\infty} C_{n} \exp [i m(n+1) \pi]|n, n\rangle \otimes\left[\cos \left(\frac{\theta}{2}\right)|\uparrow\rangle+\sin \left(\frac{\theta}{2}\right) \exp [i(\phi-m \pi)]|\downarrow\rangle\right]
$$




$$
\begin{aligned}
& \left|\psi\left(T=\frac{m^{\prime}}{2} \pi\right)\right\rangle=\sum_{n=0}^{\infty} \exp (i n \pi)\left[C_{2 n} \exp (i \phi) \sin \left(\frac{\theta}{2}\right)|2 n, 2 n\rangle\right. \\
& \left.-C_{2 n+1} \exp \left(i \frac{m^{\prime} \pi}{2}\right) \cos \left(\frac{\theta}{2}\right)|2 n+1,2 n+1\rangle\right] \otimes\left[\exp \left(-i \frac{m^{\prime} \pi}{2}\right)|\uparrow\rangle+z^{-1}|\downarrow\rangle\right],
\end{aligned}
$$

From (13) it is evident that when $T=m \pi$ the bipartite tends to its initial form, but the states of the field and atom include additional phase factors. This behavior is quite similar to that of the standard JCM [3] and two-atom JCM [47]. Nevertheless, from (14) the bipartite is disentangled, however, some information from the field is involved in the atomic system and vice versa. It seems that this is the origin in the long-lived disentanglement at these values of the interaction times. Moreover, expression (14) shows that when the atom is initially in the excited (ground) state the system can generate odd (even) two-mode squeezed vacuum state. Thus at one-half revival time the system can generate two-mode cat states, which is similar to the standard JCM [3]. We conclude this part by writing down the explicit form of the wavefunction for $(\theta, \phi, \eta)=(\pi / 2,0,0)$, which can be evaluated as

$$
|\psi(T)\rangle=\frac{1}{\sqrt{2}} \sum_{n=0}^{\infty} C_{n} \exp [i(n+1) T]|n, n\rangle \otimes[|\uparrow\rangle+|\downarrow\rangle] .
$$

From (15) it is evident that the bipartite exhibits always disentanglement, i.e. $\xi(T)=0$. From the above investigation one can notice that for $\eta=0$ the behavior of the $\xi(T)$ is consistent with that of the atomic inversion.

Now we draw the attention to the influence of the Kerr-like medium on the behavior of the $\xi(T)$ for the system under consideration (see Fig. 2(c)). The comparison between Fig. 2(a) and Fig. 2(c) shows that involving Kerr-like medium in the system drastically changes the behavior of the $\xi(T)$, e.g. it increases the amount of entanglement in the bipartite. This is related to several facts: (i) Kerr-like medium provides non-trivial photon-dependent phase in the wavefunction (cf. (5)) as well as it makes the Rabi oscillation as a nonlinear function in terms of $n$. (ii) The Kerr-like medium alone has a nonclassical nature where it can generate superposition states such as Yurke-Stoler states [48]. It is obvious that for $\eta \neq 0$ the evolutions of the $\left\langle\hat{S}_{z}(T)\right\rangle$ and $\xi(T)$ generally are not consistent.

The final remark, for the system under consideration we have found that the von Neumann entropy gives typical behavior as that of the $\xi(T)$. In the following section we study the evolution of the atomic Wehrl entropy and compare it with that of the $\xi(T)$.

\section{ATOMIC WEHRL DENSITY AND ENTROPY}

In this section we investigate the atomic Wehrl density and atomic Wehrl entropy AWE for obtaining more information on the system. Also we compare the behavior of these quantities with those given in section 3 . We start the investigation by defining the atomic $Q$-function as [49]:

$$
Q_{a}(\Theta, \Phi, T)=\frac{1}{2 \pi}\left\langle\Theta, \Phi\left|\hat{\rho}_{a}(T)\right| \Theta, \Phi\right\rangle,
$$

where $\hat{\rho}_{a}(T)$ is the atomic reduced density matrix (8) and $|\Theta, \Phi\rangle$ is the atomic coherent state expressed as

$$
|\Theta, \Phi\rangle=\cos (\Theta / 2)|\uparrow\rangle+\sin (\Theta / 2) e^{i \Phi}|\downarrow\rangle,
$$


where $0 \leq \Theta \leq \pi, 0 \leq \Phi \leq 2 \pi$. The definition (16) means that two different spin coherent states overlap unless they directed into two antipodal points on the sphere [49]. Here we shows that $Q_{a}$ can give qualitative information on the entanglement in the bipartite. From (8), (16) and (17) one can evaluate $Q_{a}(\Theta, \Phi, T)$ as

$$
Q_{a}(\Theta, \Phi, T)=\frac{1}{4 \pi}\left\{1+\rho_{z}(T) \cos \Theta+\left[\rho_{x}(T) \cos \Phi+\rho_{y}(T) \sin \Phi\right] \sin \Theta\right\}
$$

One can easily check that the $Q_{a}$ given by (18) is normalized. From (18) it is obvious that at particular values of the $\Theta$ and $\Phi$ the $Q_{a}$ can give information on the dipole moment and the atomic energy. Moreover, we have found that the behavior of the $Q_{a}(\Theta, \Phi, T)$ confirms the information obtained in the previous section. For instance, when the atom is initially in the excited or ground state and $T=m \pi$, where $m$ is integer, the $Q_{a}(\Theta, \Phi, T)$ exhibits behavior as that of the initial case. More illustratively, at these values of the interaction times we have $\rho_{z}(T)=\rho_{z}(0), \quad \rho_{x}(T)=\rho_{y}(T)=0$ and hence (18) reduces to

$$
Q_{a}(\Theta, \Phi, T=m \pi)=\frac{1}{4 \pi}\left[1+\rho_{z}(0) \cos \Theta\right] .
$$

From (19),$Q_{a}$ is independent of $\Phi$ and hence it exhibits wave shape in the $\Theta-\Phi$ plane with amplitude $\rho_{z}(0)$. On the other hand, when the atom is in the excited or ground or superposition states with $(\theta, \phi)=(\pi / 2, \pi / 2)$ and $T=m \pi / 2$, where $m$ is odd integer, the $Q_{a}(\Theta, \Phi, T)$ exhibits two-peak structure (, i.e. one peak is up and the other is down) as shown in Fig. 3a (for given values of the interaction parameters). Additionally, we plot $Q_{a}(\Theta, \Phi, T)$ for the atomic superposition state at $T=\pi$ in Fig. 3(b). The comparison between Fig. 3(a) and Fig. 3(b) shows that the locations of the maximum and minimum values in the $Q_{a}(\Theta, \Phi, T)$ are interchanged even though in these cases the bipartite is disentangled (cf. (14)). For atomic trapped case (cf. (15)) we have $\rho_{x}(T)=\rho_{x}(0), \quad \rho_{y}(T)=\rho_{z}(T)=0$ and hence (18) becomes time independent. Now we draw the attention to the influence of the $\eta$ on the behavior of the $Q_{a}(\Theta, \Phi, T)$, which is plotted in Fig. 3(c). We have generally found when the atom is in the excited or ground state and $T=m \pi$ the $Q_{a}(\Theta, \Phi, T)$ is insensitive of $\eta$, however, at $T=m \pi / 2$, e.g. for the excited atomic state, the two-peak structure involved in the $Q_{a}$ for $\eta=0$ is destroyed (compare Fig. 3(b) and Fig. 3(c)). This confirms that the bipartite is entangled (disentangled) at $T=\pi / 2(T=\pi)$. From the facts presented above and previous section we can conclude that $Q_{a}$ can give qualitative information on the entanglement in the bipartite. It is worth mentioning that the $Q$ function of the field can manifest squeezing involved in the field components, however, this is not the case for $Q_{a}$. This is related to the difference between the bosonic and fermonic algebras.

Now we draw the attention to the AWE, which is defined as [33]:

$$
W_{a}(T)=-\int_{0}^{2 \pi} \int_{0}^{\pi} Q_{a}(\Theta, \Phi, T) \ln Q_{a}(\Theta, \Phi, T) \sin \Theta d \Theta d \Phi .
$$

It is worth reminding that the definition (20) is given in analogy with that of the field Wehrl entropy [26]. Furthermore, the $W_{a}(T)$ cannot be negative as a result of the $Q_{a}$ is a nonnegative function. As it is generally difficult to find a closed form for the $W_{a}(T)$ numerical techniques have to be used. Nevertheless, at particular values of the interaction parameters the exact form can be obtained. For instance, for (19) the relation (20) can be evaluated as

$$
W_{a}(T=m \pi)=\frac{1}{2}+\ln (4 \pi)+\frac{1+\rho_{z}^{2}(0)}{4 \rho_{z}(0)} \ln \left[\frac{1-\rho_{z}(0)}{1+\rho_{z}(0)}\right]-\frac{1}{2} \ln \left(1-\rho_{z}^{2}(0)\right) .
$$



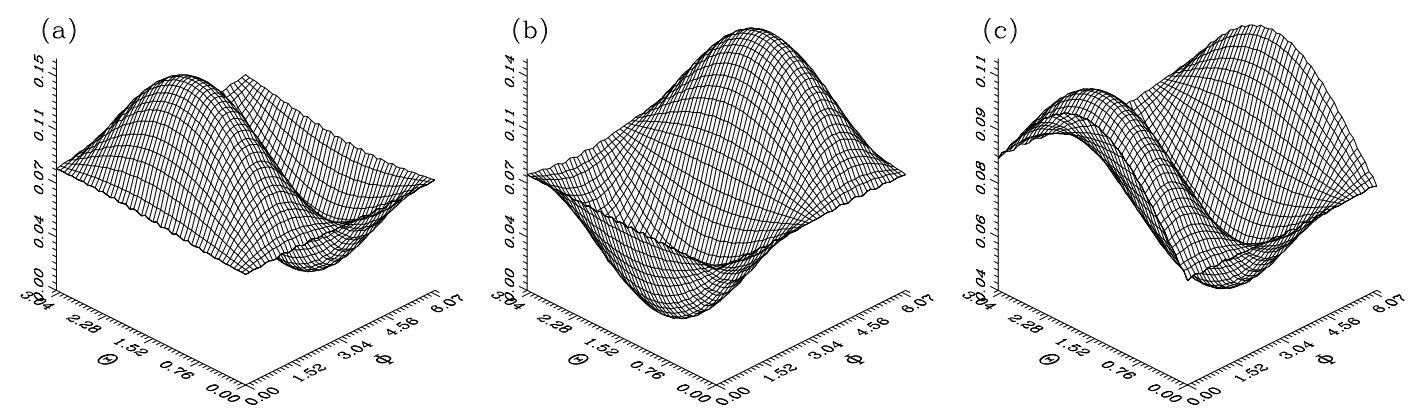

FIG. 3: The $Q_{a}(\Theta, \Phi, T)$ for $\sinh ^{2} r=10$ and $(\theta, \phi, T, \eta)=(0,0, \pi / 2,0) \quad(\mathrm{a}),(\pi / 2, \pi / 2, \pi, 0)$ (b) and $(0,0, \pi / 2,0.03)(\mathrm{c})$.
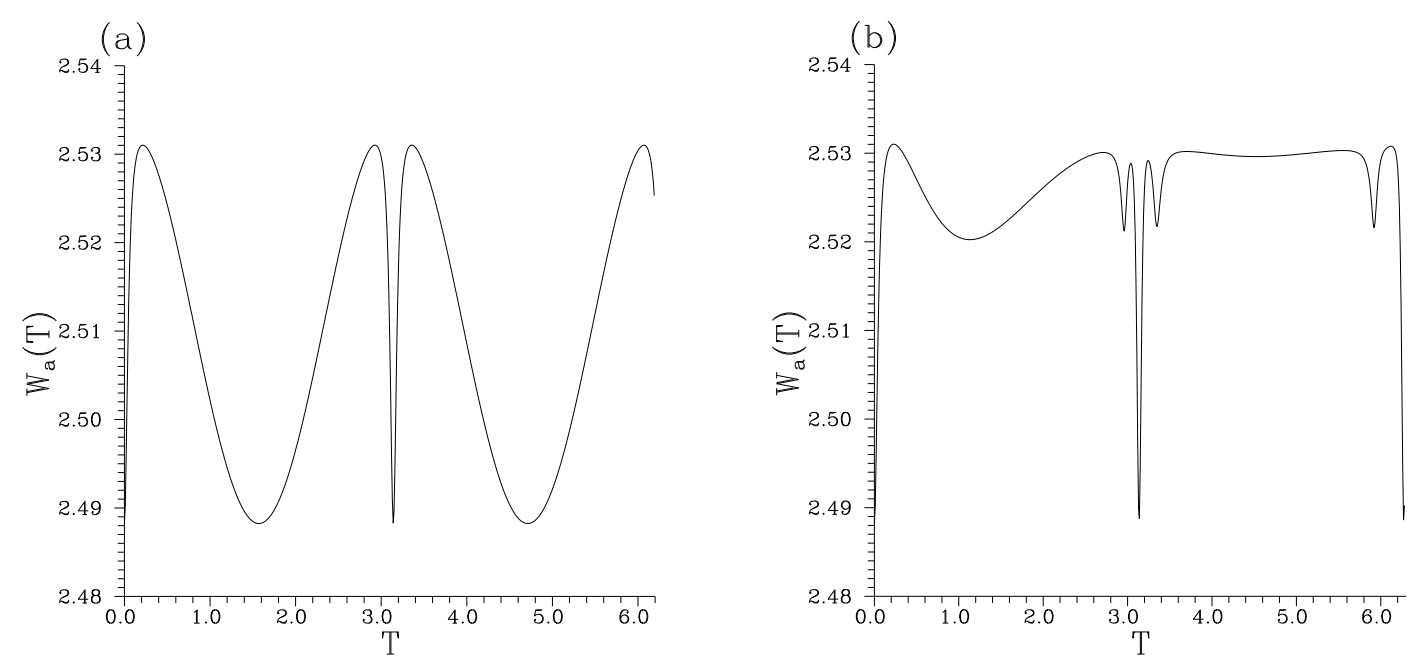

FIG. 4: The $W_{a}(T)$ versus scaled time $T$ for $\sinh ^{2} r=10$ and $(\theta, \phi, \eta)=(0,0,0)$ (a) and $(0,0,0.03)$ (b).

From expression (21) for atom initially in the excited or ground state we obtain $W_{a}(T=$ $m \pi) \simeq 2$.488. In the language of entanglement the bipartite is disentangled when $W_{a} \simeq$ 2.488. This value represents the lower bound of the $W_{a}(T)$. In Figs. 4(a) and (b) we plot $W_{a}(T)$ for the same values of the interaction parameters as in Figs. 2(a) and (c), respectively. Surprisingly one can observe that the behaviors in the Figs. 4(a) and (b) are completely similar to those in the Figs. 2(a) and (c), respectively. We have checked this fact for different values of the interaction parameters and obtained the same conclusion. The similarity between the behaviors of the $\xi(T)$ and $W_{a}(T)$ can be realized from the relations (12) and (18), i.e. the LE and the atomic Wehrl density and/or AWE are function in $\rho_{x}(T), \rho_{y}(T), \rho_{z}(T)$. Moreover, the upper bound of the AWE can be obtained by comparing the maximum value of the $\mathrm{LE}(=0.5)$ with the maximum value of the AWE $(\simeq 2.53102)$. Thus the upper bound of the AWE is 2.53102. It is obvious that the AWE is compact informative one-parameter measure describing the entanglement dynamics of the system at all values of the interaction time. From this information we can conclude that the $W_{a}(T)$ is a new measure for quantifying the entanglement in the bipartite.

The final remark is: the LE associated with the reduced atomic and reduced field density matrices can give typical information on the entanglement in the bipartite. This is not the 
case for the field Wehrl entropy $W_{f}$ and the atomic Wehrl entropy $W_{a}$. For instance, as we mentioned in the Introduction the $W_{f}$ gives information on the RCP occurred in the atomic inversion as well as on the splitting of the Husimi $Q$ function in the course of the collapse region [30]. The difference between the behaviors of the $W_{f}$ and $W_{a}$ is related to that the $Q$ functions of the field and atom provide different information on the system, where the former and the latter give information on the atomic inversion [50] and on the entanglement in the bipartite, respectively.

\section{CONCLUSION}

In this paper we have studied the properties of the TJCM (involving a Kerr-like medium) when the modes and atom are initially in the TMS and atomic superposition state, respectively. Particularly, we have investigated the atomic inversion, LE, atomic Wehrl density and AWE. We have shown that all these quantities can give information on the entanglement in the bipartite. The bipartite exhibits instantaneous and long-lived disentanglement at particular values of the interaction parameters. We have derived the explicit forms for the atomic and field states at the disentanglement times. We have shown that the atomic Wehrl entropy (density) gives quantitative (qualitative) information on the entanglement in the bipartite. Most importantly, the LE and AWE can give typical information on the entanglement in the bipartite. This is related to that these quantities can be expressed in terms of the expectation values of the atomic operators. In this regard the $W_{a}$ can be interpreted as an information measure for such system. Finally, we have shown that the Kerr-like medium increases the degree of entanglement in the bipartite.

\section{Acknowledgement}

The authors (F.A.A.E., S. A-K, M. A-A) are grateful to the Cyberspace Security Laboratory, MIMOS Berhad, Technology Park Malaysia, 57000 Kuala Lumpur, Malaysia for hospitality and financial support.

[1] Jaynes E T and Cummings F W 1963 Proc. IEEE 5189.

[2] Rempe G, Walther H and Klein N 1987 Phys. Rev. Lett. 57353.

[3] El-Orany F A A and Obada A-S 2003 J. Opt. B: Quant. Semiclass. Opt. 560.

[4] Alsingh P, Zubairy M S 1987 J. Opt. Soc. Am. B 4 177; Puri R, R.K. Bullough R K 1988 J. Opt. Soc. Am. B 5 2021; Mahmood S, Ashraf M M 1996 Opt. Commun. 132 457; Joshi A 2000 Phys. Rev. A 62043812.

[5] Buck B and Sukumar C V 1981 Phys. Lett. A 81 132; Sukumar C V and Buck B 1984 J. Phys. A 7 877; Bužek V 1989 J. Mod. Opt. 44 675; Fu C-R and Gong C-D 1997 J. Mod. Opt. 44675.

[6] Cardimona D A, Kovanis V, Sharma M P and Gavrielides A 1991 Phys. Rev. A 433710.

[7] El-Orany F A A, Mahran M H, Wahiddin M R B and Hashim A M 2004 Opt. Commun. 240 169.

[8] El-Orany F A A 2004 J. Phys. A: Math. Gen. 376157. 
[9] Gerry C C and Eberly J H 1990 Phys. Rev. A 426805.

[10] Gou S-C 1990 J. Mod. Opt. 37 1469; Ashraf N M and Razmi M S K 1992 J. Mod. Opt. 39 2245; Gou S-C 1993 Phys. Rev. A 48 3233; Iwasawa H and Matsuo 1995 Opt. Commun. 117 550; Napoli A and Messina A 1996 J. Mod. Opt. 43 649; ibid 1987353433.

[11] Cardimona D A, Sharma M P and Ortega M A 1989 J. Phys. B 22 4029; Cardimona D A 1990 Phys. Rev. A 415016.

[12] Barnett S M and Knight P L 1984 Opt. Acta 31 435; ibid 1203; Mahmood S and Zubairy M S 1987 Phys. Rev. A 35 425; Iqbal M S, Mahmood S, Razmi M S K and Zubairy M S 1988 Opt. Soc. Am. B 5 1312; Sharma M P, Cardimona D A and Gavrielides A 1989 Opt. Commun. 72 291; Sharma M P, Cardimona D A and Gavrielides A 1989 J. Opt. Soc. Am. B 61942.

[13] El-Orany F A A 2005 J. Opt. B: Quant. Semiclass. Opt. 7341.

[14] Abdel-Aty M, Abdalla M S and Obada A-S F 2002 J. Opt. B: Quant. Semiclass. Opt. 4 S133.

[15] Bennet C, Brassard G, Crepeau C, Jozsa R, Peres A and Wooters W K 1993 Phys. Rev. Lett. 701895.

[16] Benenti G, Casati G and Strini G 2005 "Principle of Quantum Computation and Information" (World Scientific, Singapore).

[17] Bennet C H, Brassard G, Crepeau C, Jozsa R, Peresand A and Wootters W K 1993 Phys. Rev. Lett. 701895.

[18] Ekert A 1991 Phys. Rev. Lett. 67 661; Cirac J I and Gisin N 1997 Phys. Lett. A 229 1; Fuchs C A, Gisin N, Griffiths R B, Niu C-S and Peres A 1997 Phys. Rev. A 561163.

[19] Ye L and Guo G-C 2005 Phy. Rev. A 71 034304; Mozes S, Oppenheim J and Reznik B 2005 Phys. Rev. A $\mathbf{7 1} 012311$.

[20] Glöckl O, Lorenz S, Marquardt C, Heersink J, Brownnutt M, Silberhorn C, Pan Q, Loock P V, Korolkova N and Leuchs G 2003 Phys. Rev. A 68 012319; Yang M, Song W and Cao Z-L 2005 Phys. Rev. A 71 034312; Li H-R, Li F-L, Yang Y and Zhang Q 2005 Phys. Rev. A 71 022314 .

[21] Wootters W K 1998 Phys. Rev. Lett. 80 2245; Hill S and Wootters W K 1997 Phys. Rev. Lett. 785022.

[22] Peres A 1996 Phys. Rev. Lett. 77 1413; Horodecki P 1997 Phys. Lett. A 232333.

[23] von Neumann J 1955 "Mathematical Foundations of Quantum Mechanics" (Princeton University Press, Princeton, NJ).

[24] Shannon C E and Weaver W 1949 "The Mathematical Theory of Communication" (Urbana University Press, Chicago).

[25] Phoenix S J D and Knight P L 1988 Ann. Phys. (N. Y.) 186381.

[26] Wehrl A 1978 Rev. Mod. Phys. 50 221; Wehrl A 1991 Rep. Math. Phys. 30119.

[27] Bužek V, Keitel C H and Knight P L 1995 Phys. Rev. A 51 2575; Vaccaro J A and Orlowski A 1995 Phys. Rev. A 51 4172; Watson J B, Keitel C H, Knight P L and Burnett K 1996 Phys. Rev. A 729.

[28] Bužek V, Keitel C H and Knight P L 1995 Phys. Rev. A 512594.

[29] Anderson A and Halliwell J J 1993 Phys. Rev. D 482753.

[30] Orlowski A, Paul H and Kastelewicz G 1995 Phys. Rev. A 521621.

[31] Miranowicz A, Matsueda H and Wahiddin M R B 2000 J. Phys. A: Math. Gen. 33 51519; A Miranowicz A, Bajer J, Wahiddin M R B and Imoto1 N 2001 J. Phys. A: Math. Gen. 34 3887.

[32] Jex I and Orlowski A 1994 J. Mod. Opt. 412301.

[33] Obada A-S and Abdel-Khalek S 2004 J. Phys. A: Math. Gen. 376573. 
[34] Ma X and Rhodes W 1990 Phys. Rev. A 41 4625; Lo C F and Sollie R 1993 Phys. Rev. A 47 733.

[35] Braunstein S L and Kimble H J 1998 Phys. Rev. Lett. 80869.

[36] Pereira S F, Ou Z Y and Kimble H J 2000 Phys. Rev. A 62 042311; Reid M D 2000 Phys. Rev. A 62 062308; Silberhorn C, Korolkova N and Leuchs G 2002 Phys. Rev. Lett. 88167902.

[37] Reid M D 1989 Phys. Rev. A 40 913; Ou Z Y, Pereira S F, Kimble H J and Peng K C 1992 Phys. Rev. Lett. 683663.

[38] Lam P K, Ralph T C, Buchler B C, McClelland D E, Bachor H A and Gao J 1999 J. Opt. B: Quant. Semiclass. Opt. 1469.

[39] Marino A M, Stroud C R Jr, Wong V, Bennink R S and Boyd R W quant-ph/0605230v1.

[40] Gou S 1989 Phys. Rev. A 405116.

[41] Scully M O 1985 Phys. Rev. Lett. 552802.

[42] Chow W W, Scully M O and Stoner J 1975 Phys. Rev. A 111380.

[43] Scully M O and Zubairy M S 1988 Opt. Commun. 66303.

[44] Caves M C, Zhu C, Milburn G J and Schleich W 1991 Phys. Rev. A 433854.

[45] Rungta P, Bužek V, Caves C M, Hillery H and Milburn G J 2001 Phys. Rev. A 64042315.

[46] Gea-Banacloche 1990 Phy. Rev. Lett. 653385.

[47] El-Orany F A A 2006 Phys. Scripta 74563.

[48] Yurke B and Stoler D 1986 Phys. Rev. Lett. 5713.

[49] Zyczkowski K 1999 quant-ph/9910088.

[50] Eiselt J and Risken H 1989 Opt. Commun. 72 351; Eiselt J and Risken H 1991 Phys. Rev. A 43 346; Eiselt J and Risken H 1991 Phys. Rev. A 44 4623; Werner M J and Risken H 1991 Quant. Opt. 3 185; Miller C A, Hilsenbeck J and Risken H 1992 Phys. Rev. A 464323. 